\title{
Response of HIV-Infected Patients with Syphilis to Therapy with Penicillin or Intravenous Ceftriaxone
}

\author{
P. Spornraft-Ragaller ${ }^{1}$, S. Abraham¹, C. Lueck², M. Meurer ${ }^{1}$ \\ ${ }^{1}$ Department of Dermatology, ${ }^{2}$ Institute of Microbiology, University Hospital Carl Gustav Carus, Technical University of Dresden, \\ Germany
}

\begin{abstract}
Background: Ceftriaxone is commonly used as an alternative antibiotic drug in treating syphilis but clinical data on its efficacy are limited. Objective: To evaluate the response of HIV-infected patients with active syphilis to treatment with penicillin or ceftriaxone. Methods: A retrospective study involving 24 consecutive patients with a positive Veneral Disease Research Laboratory test (VDRL) and at least one specific treponemal test. 12 patients were treated with different regimens of high-dose penicillin $G$ for at least 2 weeks. Another 12 patients were treated with ceftriaxone 1-2g per day intravenously for 10-21 days.

Results: After a median follow up of 18,3 months all patients of the penicillin-treated group and 11 of 12 ceftriaxone-treated patients showed a $\geq 4$-fold decline in VDRL-titers; $91 \%$ of them already within 6 months after therapy.

Conclusion: Our serological data demonstrate a comparable efficacy of currently recommened penicillin and ceftriaxone treatment regimens for active syphilis in HIV-infected patients.
\end{abstract}

Key words: Syphilis, HIV-Infection, Ceftriaxone, Penicillin

\section{INTRODUCTION}

Syphilis in HIV-infected patients is reported to show a more severe and accelerated course [1-4] with a higher risk for progression to neurosyphilis $[5,6]$. Therefore, in this population, close monitoring for neurosyphilis is recommended and in cases of latent syphilis with unknown duration, lumbar puncture should be performed. Since this procedure may be refused, in such cases high -dose parenteral therapy regimens are frequently warranted. Once neurosyphilis is excluded, current European and US-guidelines for treatment of syphilis make no distinction between patients with or without HIV-infection [7, 8]. The treatment of choice for neurosyphilis is intravenous benzyl penicillin $G$, which results in treponemicidal levels in the cerebrospinal fluid. However, the recommended administration of 3-6 doses per day often requires hospitalisation of the patients. Alternative antibiotic substances are limited; in European guidelines they include oral therapy with doxycycline, whereas the CDC favours parenteral therapy with ceftriaxone. Only few studies including a low number of predominantly HIV-infected patients with neurosyphilis or latent syphilis have shown a similar efficacy of ceftriaxone and penicillin
[9-11]. Despite of the lack of clinical evidence, ceftriaxone is commonly used as an alternative in treating syphilis [12] and therefore, more reports on its efficacy in this setting are clearly needed.

\section{PATIENTS AND METHODS}

Between January 2001 and December 2008, 29 consecutive HIV-infected patients with active syphilis were identified at the Department of Dermatology at the University Hospital, Technical University of Dresden. Diagnosis of syphilis was confirmed by a positive VDRL and at least one additional specific treponemal test (TPHA, TPPA, Treponema pallidum immunoblot, IgG- and 19S-IgM fluorescence treponema absorption-test). All 29 patients were treated but only $24 \mathrm{pa}-$ tients with one or more follow up visits were included in this study. A mean of 7.7 (1-21) serological follow up investigations for syphilis per patient were performed; data were collected until 31.5.2009.

All 24 patients were men who had sexual contacts with men (MSM) with a median age of 41 (29-57) years at the time of diagnosis of syphilis. Baseline VDRL ranged from 1:8 to1:512. 21 patients presumably had early syphilis, predominantly at stage II. 17 of 24 patients showed clinical manifestations consistent with syphilis when seen in our outpatient clinic. In 6 of 24 patients lumbar puncture was performed and in 3 patients neurosyphilis was diagnosed. In 2 patients, serology and history pointed to reinfection; in two other patients reactivation of a previous syphilis infection treated elsewhere could not be excluded, as a VDRL test prior to the current syphilis episode was not available (Table1).

12 patients with syphilis were treated with penicillin: 8 subjects received benzathine penicillin $2.4 \mathrm{MU}$ intramuscularly (i.m.) in weekly intervals for 3 weeks $(\mathrm{n}=7)$ or 2 weeks $(\mathrm{n}=1) ; 2$ patients received clemizole penicillin G 1 MU i.m. daily for 14 or 21 days and 2 patients penicillin $G$ intravenously (i.v.) 3x $10 \mathrm{MU}$ daily for 21 days. 12 patients received i.v. ceftriaxone: 8 patients $2 \mathrm{~g}$ once per day for 10-14 days, 2 patients $2 \mathrm{~g}$ for 21 days and another 2 patients $1 \mathrm{~g}$ for 14 days.

The patients were compared according to treatment with either penicillin based ( $\mathrm{n}=12$ ) or, more recently, i.v. ceftriaxone based regimens $(n=12)$. After treatment, all patients had at least one follow up-investigation of VDRL, performed between 1 and 19 months after completion of therapy. The median follow up 
time was 18.3 months (mean 29.8) for all subjects; 38.3 months for the penicillin group (mean 38.2) and 11.5 months (mean 21,8) for the ceftriaxone group ( $\mathrm{p}<$ 0.13). 7 patients in each treatment group received highly active antiretroviral therapy (HAART). The mean CD4+ T cell counts in peripheral blood in all patients were $358 / \mu \mathrm{l}$ (24-849) before treatment of syphilis.

Serological treatment response was defined as a $\geq 4$ fold decrease (or 2 dilutions) in VDRL-titer or reversion of VDRL to nonreactive. Neurosyphilis was diagnosed when a specific treponemal $\operatorname{IgG}$ production in cerebrospinal fluid (CSF) with an ITpA-Index of $\geq 4$ (based on TPPA) compared to serum was demonstrated. Syphilis stage I and II was diagnosed in patients who presented with typical symptoms in our outpatient clinic. Most of these cases were previously seronegative with documented seroconversion. Reinfection was considered when the VDRL-test rose $\geq 4$ fold after previous reversion to negative. Early latent syphilis was assumed in asymptomatic patients who had experienced symptoms consistent with syphilis less than a year before diagnosis. Patients without documented seroconversion and without history of symptoms were classified as latent syphilis of unknown duration.

For statistical analysis the two sided Mann Whitney U-test for unpaired samples and Fisher's Exact test were performed (SPSS, Version 17).

\section{RESULTS}

24 patients were analyzed; 12 had received penicillin in various dose regimens mainly i.m. and 12 had been treated with i.v. ceftriaxone at daily doses of $2 \mathrm{~g}$ in most cases.

Comparing both treatment groups, the patients did not differ in respect to age $(\mathrm{p}=0.38)$ or the proportion receiving HAART. Cases with symptomatic syphilis were slightly more common in the penicillin group reflecting a greater proportion of primary and secondary syphilis. Overall median baseline VDRL was $1: 64(1: 8-1: 512)$ with a tendency to higher titers in the ceftriaxone group (median 1:64-1:128) vs. $1: 32$ in the penicillin group ( n.s., $p=0.23$ ). The patients of the ceftriaxone group had higher baseline CD4-T-cell counts but the difference did not reach statistical significance $(p=0.08)$. Six cases treated with i.m. penicillin could clearly be diagnosed as early syphilis with documented seroconversion or reinfection, whereas ceftriaxone or i.v. penicillin was chosen for treatment of patients without documented seroconversion ( $\mathrm{n}=$ 8 ; $67 \%$ of the patients in the ceftriaxone group) or in cases with suspected penicillin allergy $(n=3)$. Herxheimer's reactions occurred in some patients with secondary syphilis but were not monitored consistently, because patients who refused hospitalisation were given prophylaxis (50 $\mathrm{mg}$ prednisolone).

Table 1. Baseline characteristics.

\begin{tabular}{|c|c|c|c|}
\hline \multirow{2}{*}{$\begin{array}{l}\text { Characteristic } \\
\text { Age, median years }\end{array}$} & $\begin{array}{l}\text { Penicillin } \\
\mathrm{n}=12\end{array}$ & $\begin{array}{l}\text { Ceftriaxone } \\
\mathrm{n}=12\end{array}$ & \multirow{2}{*}{$\begin{array}{l}\mathrm{p} \\
0,38\end{array}$} \\
\hline & $42 \quad(33-57)$ & $40.5(29-47)$ & \\
\hline Follow-up, median months & $38,3 \quad(5.5-73)$ & $11.5(1.5-78.5)$ & 0.13 \\
\hline CD $4+T$ cell counts, median cells $/ \mu \mathrm{l}$ & $264 \quad(128-849)$ & $411 \quad(24-707)$ & 0.08 \\
\hline HAART, $\mathrm{n}=$ & 7 & & \\
\hline Baseline VDRL, median & $1: 32$ & $1: 64-1: 128$ & 0.23 \\
\hline Early syphilis & 11 of $12(92 \%)$ & 9 of $12(75 \%)$ & 0.59 \\
\hline stage I & 2 & 1 & \\
\hline stage II & 6 & 6 & \\
\hline Early latent & 3 & 2 & \\
\hline Neurosyphilis $\quad \mathrm{n}=$ & 1 & 2 & \\
\hline Probably late latent, unknown duration, $\mathrm{n}=$ & 0 & 1 & \\
\hline Clinical signs at presentation, $\mathrm{n}=$ & 9 of $12(75 \%)$ & 7 of $12(58 \%)$ & 0.66 \\
\hline Reinfection, $\mathrm{n}=2$ & 0 & & \\
\hline possible reactivation, $\mathrm{n}=$ & 0 & 2 & \\
\hline documented seroconversion, $\mathrm{n}=$ & 6 of $12(50 \%)$ & 4 of $12(33 \%)$ & 0.68 \\
\hline lumbar puncture, $\mathrm{n}=$ & 2 & 4 & \\
\hline
\end{tabular}

Follow-up AND Treatment Results

Table 2. Treatment results.

\begin{tabular}{|c|c|c|c|}
\hline & $\begin{array}{l}\text { Penicillin } \\
\mathrm{n}=12\end{array}$ & $\begin{array}{l}\text { Ceftriaxone } \\
\mathrm{n}=12\end{array}$ & $\mathrm{p}$ \\
\hline \multirow{5}{*}{$\begin{array}{l}\geq 4 \text {-fold decrease in VDRL-titer } \begin{array}{l}\text { after treatment } \\
\text { within } 3 \text { months } \\
\text { within } 6 \text { months } \\
\text { within } 12 \text { months } \\
\text { within } 20 \text { months }\end{array}\end{array}$} & 12 of 12 & 11 of 12 & \\
\hline & 5 of $7(71 \%)$ & 10 of $11(91 \%)$ & 0.52 \\
\hline & 9 of $10(90 \%)$ & & \\
\hline & 11 of 11 & 11 of $12(92 \%)$ & \\
\hline & 12 of 12 & & \\
\hline Negative VDRL-titer at the end of follow up & 6 of 12 & 4 of 12 & 0.68 \\
\hline
\end{tabular}


The median overall follow-up time was 18,3 months. A first control of VDRL within 3 months after completion of therapy was available in 11 cases of the ceftriaxone group and in 7 patients of the penicillin group.10 of 11 ceftriaxone treated patients showed a $\geq 4$ fold decrease of VDRL-titers within 3 months (median 1,75 months) as well as 5 of 7 cases of the penicillin group. When the VDRL test was performed within 6 months after therapy, the percentage of patients with a 4-fold VDRL decrease rose to $90 \%$ in the penicillin group ( 9 of 10 cases). In the remaining patients, a first VDRL control was available only at 6,5, 7,5, 11 and 19 months after therapy. At that time, these patients showed already a reversion of the VDRL to negative $(n=3)$ or weakly positive (titer 1:2; $\mathrm{n}=1$ ). Within a year after therapy, 22 of 23 patients showed a serological response to treatment with either penicillin or ceftriaxone and taken together, 23 of 24 patients responded within 20 months. 10 patients achieved a negative VDRL; 7 in the penicillin group after a median follow-up of 38,3 months and 3 in the ceftriaxone group after a median follow up of 11,5 months. We additionally analyzed 13 patients with a VDRL being available at approximately one year after therapy (13 months $\mathrm{n}=4 ; 12$ months $\mathrm{n}=5 ; 11$ months $n=2 ; 10$ months $n=2) .7$ patients were treated with penicillin and 6 patients with ceftriaxone. At that time, all patients showed $\mathrm{a} \geq 8$ fold (or 3 dilution) decline of VDRL or reversion of the VDRL to negative. The latter occurred in 3 patients with a VDRL being available after 13 months and in 1 patient after 10 months, respectively. Only one patient with presumably latent neurosyphilis, assigned to the ceftriaxone group, remained serofast after treatment with either i.v. ceftriaxone $2 \mathrm{~g}$ for 14 days or subsequent i.v. penicillin for 3 weeks. This patient had been newly diagnosed to have both HIV- and syphilis infection at CD4-T-cell counts of $24 / \mu$ l. Initial VDRL was $1: 16$ and TPHA was 1:640 without demonstration of specific IgM (19-S IgM-FTA-ABS-Test). A previous syphilis infection was not known. There were no clinical signs of neurosyphilis and no pleocytosis of the cerebrospinal fluid (CSF). However, the ITpA-Index was 4,7 and the TPHA-Index was 385 compared to serum (TPHA-Index of 100-500: neurosyphilis probable), thus indicating specific intrathecal IgG production. After treatment with ceftriaxone and stabilisation of CD4-T-cell counts at 322/ $\mu$ l under HAART, the second treatment with penicillin $G$ did not significantly influence VDRL- or TPHA-titers.

Most of the patients $(n=18)$ could be closely monitored with 3 or more serological follow-up data being available. Until the end of follow up, no relapse of syphilis was identified in any of the patients. However, in 2009 two patients acquired an additional syphilis infection with significant rise of specific treponemal and VDRL titers. One patient had been previously treated with penicillin and the other one with ceftriaxone. In both patients, previous VDRL titers had declined to 1 : 2 or to nonreactive, respectively. One patient showed syphilis stage I and gave a history of unprotected sex 4 weeks before development of genital ulcers; the other patient exhibited a typical exanthema of syphilis stage II. Therefore, these additional episodes of syphilis were regarded as reinfections and were not included in this study.

\section{DisCUSSION}

In concurrent HIV-infection, syphilis may show a more severe course, a more rapid progression to neurosyphilis and higher rates of treatment failure [4, 6, 13]. The magnitude of this risk is not precisely defined. There are some reports on treatment failure after therapy of early syphilis with benzathine penicillin [14]. A large randomized, prospective controlled trial in 541 patients including 101 with HIV-infection assessed enhanced therapy of early syphilis with benzathine penicillin 2,4 MU i.m. plus amoxicillin and probenecid compared to benzathine penicillin alone. This study was not able to demonstrate an advantage of the enhanced therapy $[15,16]$. Therefore, most guidelines for treatment of syphilis in HIV -infected patients do not substantially differ from those for patients without HIV-infection [16]. However, some experts recommend more intensive therapeutic regimens even for early syphilis in HIV-infection [7] with benzathine penicillin 2,4 MU in weekly intervals at day 1,8 and 15 which is in concordance with current European and German Guidelines [8, 17]. The same regimen can be used for late latent syphilis or syphilis of unknown duration if CSF examination is normal. If neurosyphilis is suspected but lumbar puncture is refused, treatment with aqueous i.v. penicillin $G$ is advisable. Alternative substances for treating neurosyphilis are scarce: In case of penicillin allergy, according to European guidelines oral therapy with doxycycline is considered; the CDC [7] in this situation proposes penicillin desensitization or ceftriaxone at a dose of $2 \mathrm{~g}$ per day i.m. or i.v. However, clinical data on the efficacy of ceftriaxone for treatment of syphilis compared to standard therapy with penicillin are limited.

In our study, HIV-infected patients with syphilis were treated with either penicillin or ceftriaxone at relatively high doses based on current treatment guidelines described above. In 24 patients we could observe a comparable effect of both penicillin and ceftriaxone based regimens after a median follow up of 18,3 months. All 12 patients treated with penicillin showed the same treatment response with a $\geq 4$-fold decline in VDRL-tests as 11 of 12 patients treated with ceftriaxone. After completion of treatment with ceftriaxone, 10 of 11 patients (91\%) achieved a $\geq 4$-fold decline in VDRL-tests already within 3 months and another patient within 6,5 months. Taken together, 11 of 12 patients $(92 \%)$ responded to therapy with ceftriaxone after 12 months except for one patient who remained serofast. After treatment with penicillin, only 5 of 7 patients showed $\mathrm{a} \geq 4$-fold decline of VDRL within 3 months. After a follow up of 19,5 months, all 12 patients treated with penicillin had shown a response to therapy. Although most patients achieved treatment response less than 12 months after completion of therapy, in 13 patients ( 7 treated with penicillin and 6 treated with ceftriaxone) the response at approximately one year (10-13 months) after therapy was determined. In all patients, $a \geq 8$ fold decline of VDRL could be observed, thus also indicating similar efficacy 
of both antibiotics. There may be a trend to a more rapid response to ceftriaxone compared to penicillin; however, despite of the lack of significant differences in baseline characteristics, a direct comparison is difficult: On one hand, the patients in the ceftriaxone group had somewhat higher baseline VDRL-titers together with higher baseline CD4-T-cell counts and therefore might have had a more rapid decrease of VDRL ; on the other hand, in this group were also more patients with unknown duration of syphilis and a more complicated disease. One patient with probably late latent neurosyphilis did not respond to treatment with either ceftriaxone or subsequent penicillin.

There exist only few small studies on clinical data concerning the efficacy of ceftriaxone for treatment of syphilis, as recently reviewed by Parkes and Stoner $[15,18]$. In a report of Smith et al. [9] 24 HIV-infected patients with asymptomatic syphilis and a rapid plasma reagin $(\mathrm{RPR})$ titer of $\geq 1: 4$ were randomized to treatment with either ceftriaxone $1 \mathrm{~g}$ i.m. $(\mathrm{n}=10)$ or enhanced treatment with procaine penicillin plus probenecid for 15 days $(n=14)$ and were followed up prospectively (median follow up 32 months for penicillin and 18 months for ceftriaxone): In this study, only $70 \%$ of procaine penicillin treated patients and $71 \%$ of ceftriaxone treated patients showed $\mathrm{a} \geq 4$ fold decline in RPR-titers. However, most of the patients were believed to have late latent syphilis and all were enrolled before the availability of HAART. In another study of the pre-HAART-era on the efficacy of ceftriaxone 1-2 g per day for 10-14 days in HIV-infected patients with latent or neurosyphilis there was a response rate of $65 \%$ in 28 patients treated with ceftriaxone vs. $62 \%$ in patients treated with benzathine penicillin [11] . A more recent study of Marra et al. [10] found that virtually all of 59 predominantly HIV-infected patients with neurosyphilis showed a normalisation of CSF white blood cell counts and CSF VDRL after various treatment regimens including ceftriaxone $2 \mathrm{~g}$ i.v. in 7 patients. In a previous randomized pilot study by the same author [19] evaluating ceftriaxone or penicillin $G$ for treatment of neurosyphilis in $30 \mathrm{HIV}$-infected patients, ceftriaxone was found to be equally effective in improving CSF markers for neurosyphilis and seemed to be superior concerning decline of serum RPR titers $(80 \%$ vs. $13 \%$ of the patients). Follow up, however, was short (3-6 months) in this study and most of the patients had a history of previous syphilis of which neurosyphilis was prevailing in the penicillin group. Another randomized study of 28 presumably HIV-negative patients with primary or secondary syphilis compared treatment with ceftriaxone i.m. or penicillin $G$ i.m. daily for 2 weeks and found no difference of clinical and serological response. After a maximum follow up of 12 months all patients achieved at least a 2-fold dilution decrease in VDRL-titers [20]. The efficacy of ceftriaxone for treatment of syphilis is also demonstrated by a recent report on 3 cases of HIV-infected patients with neurologic syphilis after treatment of primary or secondary syphilis with benzathine penicilline who responded to subsequent therapy with ceftriaxone [21]. In summary, a review of the literature suggests that ceftriaxone i.v. or i.m. seems to be similarly effective for treatment of syphilis as penicillin based regi- mens which is in concordance with our findings. There seems to be more experience with ceftriaxone in HIVcoinfected patients. The higher failure rates previously reported might be attributable to the unavailability of HAART in some of the earlier studies and to the stage of syphilis irrespective of the treatment regimen: In asymptomatic patients with late latent syphilis, reinfection or syphilis of unknown duration and low titers of VDRL, serologic follow up is difficult. Since most of our patients had early stages of syphilis, some of them with documented seroconversion, this might explain the favourable overall response to both ceftriaxone and penicillin. Limitations of our investigation are - as in some previous studies - the small sample size and the heterogeneity of active syphilis stages. Patients were treated with relatively high, but different doses of both antibiotics and follow up was retrospective and not standardized. Although not significant, patients treated with ceftriaxon had higher baseline CD4 cell counts and therefore a lower risk of neurosyphilis. However, in our experience ceftriaxone, also because of its more convenient applicability once per day, is a suitable alternative treatment agent for syphilis especially in HIV infected patients in whom parenteral therapy seems warranted more frequently. However, further prospective studies are needed to confirm these findings.

\section{REFERENCES}

1. Johns DR, Tierny M, Felsenstein D. Alterations in the natural history of neurosyphilis by concurrent infection with the human immundeficiency virus. $\mathrm{N}$ Engl J Med 1987;316:1569-72.

2. Gregory N, Sanchez M, Buchness MR. The spectrum of syphilis in patients with human immunodeficiency virus. Am Acad Dermatol 1990;22:1061-7.

3. Körber A, Dissemond J, Hillen U, Esser S. HIV- positive patient with multiple ulcerations. Hautarzt 2003;11:1098102.

4. Schöfer H, Imhof M, Thoma-Greber E, Brockmeyer NH, Hartmann M, Gerken G, et al. Active syphilis in HIV infection: a multicentre retrospective survey. The German AIDS Study Group (GASG). Genitourin Med 1996 Jun 10;72(3):176-81.

5. Gordon SM, Eaton ME, George R, et al. The response of symptomatic neurosyphilis to high-dose intravenous penicillin $G$ in patients with human immuno-deficiency virus infection. N Engl J Med 1994;331:1469-73.

6. Musher DM, Hamill RJ, Baughn RE. Effect of human immunodeficiency virus (HIV) infection on the course of syphilis and on the response to treatment. Ann Intern Med 1990;113(11):872-81.

7. Center of Disease Control. CDC genital ulcers-STD treatment guidelines.

www cdc gov/STD/treatment/2006/genital-ulcers htm 2006

8. Goh BT, van Voorst Vader PC. European guideline for the management of syphilis. Int J STD \& AIDS 2001; 12(3):14-26.

9. Smith NH, Musher DM, Huang DB, Rodriguez PS, Dowell ME, Ace W, et al. Response of HIV-infected patients with asymptomatic syphilis to intensive intramuscular therapy with ceftriaxone or procaine penicillin. Int J STD \& AIDS 2004;15:328-32.

10. Marra CM, Maxwell CL, Tantalo L, Eaton M, Rompalo AM, Raines C, et al. Normalization of cerebrospinal fluid abnormalities after neurosyphilis therapy: Does HIV status matter? Clin Infect Dis 2004;38:1001-6. 
11. Dowell E, Ross PG, Musher DM, Cate TR, Baughn RE. Response of latent syphilis or neurosyphilis to ceftriaxone therapy in persons infected with human immunodeficiency virus. Am J Med 1992;93(5):481-8.

12. Augenbraun M. Treatment of Syphilis 2001: Nonpregnant adults. Clin Infect Dis 2002;35(2):187-90.

13. Musher DM. Syphilis, neurosyphilis, penicillin and AIDS. J Infect Dis 1991;163:1201-6.

14. Berry CD, Hooton TM, Collier AC, Lukehart SA. Neurological relapse after benzathine penicillin therapy for secondary syphilis in a patient with HIV infection. $\mathrm{N}$ Engl J Med 1987;316:1587-9.

15. Parkes R, Renton A, Meheus A, Laukamm-Josten U. Review of current evidence and comparison of guidelines for effective syphilis treatment in Europe. Int J STD \& AIDS 2004;15:73-88.

16. Rolfs RT, Joesoef MR, Hendershot EF, Rompalo AM, Augenbraun MH, Chiu M, et al. A randomized trial of enhanced therapy for early syphilis in patients with and without human immunodeficiency virus infection. The Syphilis and HIV Study Group. N Engl J Med 1997; 337(5):307-14.

17. German Society of Sexually Transmitted Diseases. Diagnosis and therapy of syphilis 2008. http://www.uni-duesseldorf.de/WWW/AWMF/II/059-002 htm

18. Stoner BP. Current controversies in the management of adult syphilis. Clin Infect Dis 2007;44(3):130-46.
19. Marra CM, Boutin P, McArthur JC, Hurwitz S, Simpson PA, Haslett JA, et al. A pilot study evaluating ceftriaxone and penicillin $G$ as treatment agents for neurosyphilis in human immunodeficiency virus-infected individuals. Clin Infect Dis 2000;30(3):540-4.

20. Schöfer H, Vogt HJ, Milbradt R. Ceftriaxone for the treatment of primary and secondary syphilis. Chemotherapy 1989;35:140-5.

21. Walter T, Lebouche B, Miailhes P, Cotte L, Roure C, Schlienger I, et al. Symptomatic relapse of neurologic syphilis after benzathine penicillin $G$ therapy for primary or secondary syphilis in HIV-infected patients. Clin Infect Dis 2006;43:787-90.

Received: May 11, 2010 / Accepted: November 5, 2010

Address for correspondence:

Dr. med. Petra Spornraft-Ragaller

Dept. of Dermatology

University Hospital Carl Gustav Carus

Technical University of Dresden

Fetscherstr. 74

01307 Dresden

Germany

Phone: $++49 / 351-4583878$

Fax: $\quad++49 / 351-4585373$

E-mail: Petra.Spornraft-Ragaller@uniklinikum-dresden.de 